\title{
Structure of the superconducting state in a fully frustrated wire network with dice lattice geometry
}

\author{
S. E. Korshunov ${ }^{1,2}$ and B. Douçot ${ }^{2}$ \\ ${ }^{1}$ L. D. Landau Institute for Theoretical Physics, Kosygina 2, Moscow 119334, Russia \\ ${ }^{2}$ Laboratoire de Physique Théorique et Hautes Energies, CNRS UMR 7589, \\ Université Paris VI and VII, 4 place Jussieu, 75252 Paris Cedex 05, France
}

(Dated: October 22, 2004)

\begin{abstract}
The superconducting state in a fully frustrated wire network with the dice lattice geometry is investigated in the vicinity of the transition temperature. We express the projection of the GinzburgLandau free energy functional on its unstable subspace in terms of variables defined on the triangular sublattice of sixfold coordinated sites. For the resulting effective model, we construct a large class of degenerate equilibrium configurations, which are in one to one correspondence with ground states of the fully frustrated $X Y$ model with a dice lattice. The entropy of this set of states is proportional to the linear size of the system. Finally, we show that magnetic interactions between currents provide a degeneracy lifting mechanism and find the structure of the periodic state selected by these interactions.
\end{abstract}

PACS numbers: 74.81.Fa, 64.60.Cn, 05.20.-y

\section{INTRODUCTION}

The concept of frustration has been a common link among various problems in statistical mechanics for the past two decades at least. Even in the absence of disorder, it often results in a phenomenon of competition between several degenerate ground-states. Superconducting wire networks provide a very appealing class of systems where many subtle effects induced by frustration can be observed experimentally and analyzed theoretically [1, 2, 3, 4, 5, 6, 7, 8, 9].

For simple regular networks, a natural parameter characterizing the strength of the frustration is the ratio $f=\Phi / \Phi_{0}$ where $\Phi$ is the external magnetic flux through an elementary plaquette of the lattice and $\Phi_{0}=h c / 2 e$ is the superconducting flux quantum. For an ideal network of very thin wires, all physical properties are expected to be periodic functions of $f$, all integer values being equivalent. In this case, the maximal frustration is obtained when $f$ reaches half-integer values. Such fluxes are interesting because already for a single loop, they provide two equivalent ground states, distinguished by the orientation of the supercurrent flowing around the loop. For more complex geometries, two adjacent loops (sharing a common link) have a lower free energy when the currents in them flow in opposite directions. The possibility to fulfill this requirement for any pair of adjacent loops is a geometrical property of a given lattice, which allows one to be sure about the structure of the superconducting state without any additional analysis. This clearly holds for a square lattice [10] (where vortices of alternating signs form a regular checkerboard pattern) or for a triangular lattice.

In recent years, network geometries which do not satisfy this criterion have received a lot of attention. The most studied examples are the honeycomb 11, 12, 13, 14, 15], the kagome 14, 15, 16, 17, 18, 19, 20, 21], and the dice [22, 23, 24, 25, 26, 27] lattices. On the honeycomb lattice, the discrete degeneracy of the classical ground states in fully frustrated superconducting wire networks or Josephson junction arrays can be described in terms of formation of zero-energy domain walls in parallel to each other [13], the residual entropy of such system being proportional to its linear size. Experimentally, a cusp-like local maximum in the superconducting transition temperature $T_{c}$ is observed as the external magnetic field is varied around the value corresponding to $f=1 / 2$ [14]. This behavior has been interpreted as an evidence for a degeneracy lifting mechanism which selects a commensurate ordered pattern of vortices [14].

For the kagome lattice, the residual entropy of classical ground states is much larger, since it is proportional to the whole network area [17, 28]. The experimental situation at $f=1 / 2$ is not as clearcut as for the honeycomb lattice, since the shape of $T_{c}$ versus magnetic field curves near $f=1 / 2$ depends on the resistive criterion chosen to determine $T_{c}$ [19], or on the superconducting metal (aluminium versus niobium for instance 14]). Theoretically, various degeneracy lifting mechanisms have been studied in detail by Park and Huse [20].

On the dice lattice (see Fig. (1), the residual entropy is proportional to the system linear size [26], as for the honeycomb lattice. Experimentally, magnetic decoration experiments 24, 25] have found a highly disordered vortex pattern, with a vortex correlation length comparable to the lattice spacing. Numerical simulations [27] of the corresponding $X Y$ model support the picture proposed in Ref. 26 for the ground states, but also demonstrate, at low temperatures, an unusually slow relaxation of energy, as well as aging of phase correlation functions.

In this article we consider maximally frustrated superconducting networks on a dice lattice, in the immediate vicinity of the superconducting transition temperature. In this limit, the amplitude of the superconducting orderparameter is not necessarily uniform, and it is appropriate to use a generalization of the approach introduced by 
Abrikosov [29] in his first prediction of vortex lattices in type II superconductors. The main idea underlying this approximation is that at $T_{c}(H)$, only a small fraction of the eigenmodes of the linearized Ginzburg-Landau equations become unstable. Abrikosov developed a variational procedure where the superconducting order-parameter is constrained to remain in this unstable subspace (for $T \rightarrow T_{c}(H)$ this procedure is asymptotically exact). Minimizing the quartic term in the Ginzburg-Landau free energy functional yields then periodic vortex lattice solutions.

Adopting this approach to a dice lattice network is quite interesting since the corresponding eigenmodes (for $f=1 / 2$ ) have unusually high degeneracy and exhibit the unexpected property of an extreme form of spacial localization. It is indeed possible to construct an eigenfunction basis for which each member is non-vanishing only on a finite cluster 22, 30]. This phenomenon arises from the Aharonov-Bohm interference effect which is magnified in the geometry of the dice lattice, and these AharonovBohm cages have been evidenced experimentally by the observation of magnetoresistance oscillations in ballistic semiconductor networks [31] with the flux period $h c / e$ per elementary loop.

The main result of the present study is that in maximally frustrated superconducting wire network nonlinear effects select a class of order-parameter configurations in one to one correspondence with the ground states of the fully frustrated $X Y$ model with the same geometry [26], which may be viewed as a low temperature approximation for the Ginzburg-Landau model ignoring amplitude variations (London limit). However, the inclusion into analysis of the magnetic energy leads to the removal of the accidental degeneracy and selection of one of the periodic states minimizing the Ginzburg-Landau free energy. The same state has the lowest free energy also at lower temperatures (down to London limit), as well as in Josephson junction arrays with the same geometry.

In sections [I and [II] we express the Ginzburg-Landau functional for a fully frustrated dice lattice wire network

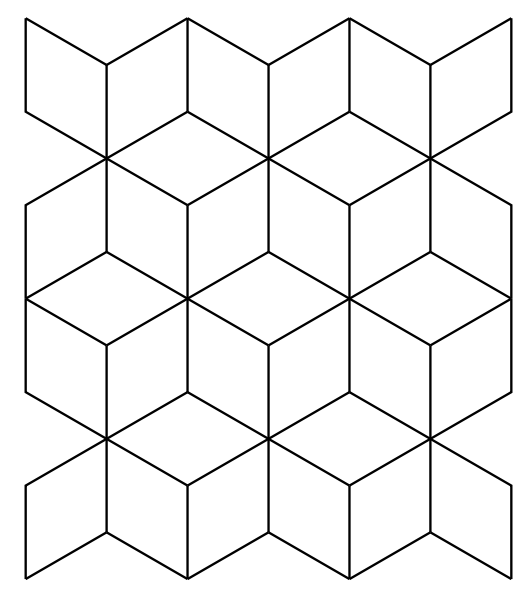

FIG. 1: Finite cluster with the dice lattice geometry. after projection on the subspace of unstable modes in terms of complex variables defined on the triangular sublattice of sixfold coordinated sites. Section IV describes the construction of periodic equilibrium states for this effective problem, and their extension to a larger class of degenerate states whose precise connection with those proposed for the corresponding $X Y$ model is established. Finally, section $\nabla$ investigates a degeneracy lifting due to magnetic interaction between currents.

\section{HARMONIC CONTRIBUTION TO FREE ENERGY}

\section{A. A single wire}

In the framework of the Ginzburg-Landau approximation the free energy of a thin superconducting wire, $F_{\text {wire }}^{\mathrm{GL}}$, can be written as the sum of the two terms,

$$
\begin{aligned}
F_{\text {wire }}^{(2)}= & \int_{0}^{L} d x\left\{-\frac{\alpha}{2}|\Delta(x)|^{2}+\right. \\
& \left.+\frac{\gamma}{2}\left|\left[-i \frac{\partial}{\partial x}-\frac{2 \pi}{\Phi_{0}} A_{\|}(x)\right] \Delta(x)\right|^{2}\right\}
\end{aligned}
$$

and

$$
F_{\text {wire }}^{(4)}=\frac{\beta}{4} \int_{0}^{L} d x|\Delta(x)|^{4},
$$

describing, respectively, the harmonic and the fourthorder contributions to $F_{\text {wire }}^{\mathrm{GL}}$. Here $\alpha \propto T_{c 0}-T, \beta$ and $\gamma$ are the coefficients of the Ginzburg-Landau expansion, $L$ is the length and $T_{c 0}$ the mean field transition temperature of the wire, $\Delta(x)$ is the superconducting order parameter as a function of the coordinate $x$ along the wire, $A_{\|}(x)$ is the projection of the vector potential on the wire and $\Phi_{0}=h c / 2 e$ is the superconducting flux quantum.

At the point of phase transition $|\Delta(x)| \rightarrow 0$ and $F_{\text {wire }}^{(4)}$ can be neglected in comparison with $F_{\text {wire. }}^{(2)}$. For the given values of $\Delta(x)$ at the ends of the wire,

$$
\Delta(0)=\Delta_{0}, \Delta(L)=\Delta_{1},
$$

the minimum of $F_{\text {wire }}^{(2)}$ is achieved when [2]

$\Delta(x)=\left[\Delta_{0} \sin \frac{L-x}{\xi}+\Delta_{1} \sin \frac{x}{\xi} \exp \left(-i A_{01}\right)\right] \frac{\exp i a(x)}{\sin \eta}$,

where $\eta=L / \xi$,

$$
\xi \equiv \xi(T)=\sqrt{\frac{\gamma}{\alpha}} \approx \frac{\bar{\xi}}{\sqrt{1-T / T_{c 0}}}
$$

is the temperature dependent correlation length [here $\bar{\xi} \sim \xi(T=0)]$, the function $a(x)$ is defined by the integral

$$
a(x)=\frac{2 \pi}{\Phi_{0}} \int_{0}^{x} d x^{\prime} A_{\|}\left(x^{\prime}\right),
$$


whereas $A_{01}$ is the value of this integral for the whole wire, $A_{01}=a(L)$.

Substitution of Eq. (4) into the expression for the superconducting current in the wire,

$$
I(x)=\frac{2 e}{\hbar} \gamma \operatorname{Re}\left[\Delta^{*}(x)\left(-i \frac{\partial}{\partial x}-\frac{2 \pi}{\Phi_{0}} A_{\|}\right) \Delta(x)\right],
$$

shows that the value of the current is constant along the wire and is given by

$$
I_{01}=-\frac{2 e}{\hbar} \frac{\gamma}{\xi \sin \eta} \operatorname{Im}\left[\Delta_{0} \Delta_{1}^{*} e^{i A_{01}}\right] .
$$

On the other hand substitution of Eq. (4) into Eq. (10) gives a simple quadratic form of $\Delta_{0}$ and $\Delta_{1}[7]$ :

$F_{\text {wire }}^{(2)}\left(\Delta_{0}, \Delta_{1}, A_{01}\right)=F_{2} \cdot\left[\cos \eta\left(\left|\Delta_{0}\right|^{2}+\left|\Delta_{1}\right|^{2}\right)-\left\langle\Delta_{0} \mid \Delta_{1}\right\rangle\right]$

where $F_{2}=\gamma /(2 \xi \sin \eta)$ and

$$
\left\langle\Delta_{j} \mid \Delta_{k}\right\rangle=\Delta_{j} \Delta_{k}^{*} e^{i A_{j k}}+\Delta_{j}^{*} \Delta_{k} e^{-i A_{j k}} .
$$

\section{B. An arbitrary network}

The function $F_{\text {wire }}^{(2)}$ defined by Eq. (9) can be then used to express the harmonic part of a free energy of a superconducting wire network $F_{\mathrm{nw}}^{(2)}$ in terms of the values of the superconducting order parameter $\Delta_{j}$ in its nodes $j$,

$$
F_{\mathrm{nw}}^{(2)}=\sum_{(j k)} F_{\mathrm{wire}}^{(2)}\left(\Delta_{j}, \Delta_{k}, A_{j k}\right) .
$$

Here the summation is performed over all links $(j k)$ of a network. In the following we assume that all the links are identical and, therefore, the function $F_{\text {wire }}^{(2)}\left(\Delta_{j}, \Delta_{k}, A_{j k}\right)$ is the same for all the links.

In the case of a network formed by identical plaquettes it is convenient to express the value of perpendicular external magnetic field $H$ in terms of the number of flux quanta per single plaquette: $f=H S / \Phi_{0}$ (here $S$ is the area of a plaquette). Then the directed summation of the variables $A_{j k} \equiv-A_{k j}$ along the perimeter of each plaquette in positive direction (denoted below as $\sum_{\square}$ ) should give

$$
\sum_{\square} A_{j k}=2 \pi f .
$$

From the form of Eq. (9) it is evident that the shift of $f$ by an integer or its reflection with respect to $f=1 / 2$ $(f \rightarrow 1-f)$ do not change the form of the expression for free energy (or can be taken care of by a redefinition of variables), and, therefore, it is sufficient to analyze the interval $0 \leq f \leq 1 / 2$. By the analogy with frustrated $X Y$ models 10] a network with the maximal irreducible value of $f$, that is with $f=1 / 2$, can be called a fully frustrated network.
When fluctuations are completely neglected, the magnetic field dependence of the superconducting transition temperature in a network $T_{c}(f)$ can be found by looking when (with the decrease of temperature) the quadratic form defined by Eqs. (9)-(11) looses its positiveness. To this end one has to analyze the system of equations obtained by the variation of $F_{\mathrm{nw}}^{(2)}$ with respect to $\Delta_{k}^{*}$,

$$
\sum_{j=j(k)}\left[\Delta_{k} \cos \eta-\Delta_{j} e^{i A_{j k}}\right]=0,
$$

where $j(k)$ are the nodes connected with $k$ by the links of a network (in the following we call them the nearest neighbors of $k$ ). The same equations can be derived [1, 2] directly in the framework of the continuous description without explicit calculation of $F_{\text {wire }}^{(2)}\left(\Delta_{j}, \Delta_{k}, A_{j k}\right)$. Multiplication of Eq. (13) by $\Delta_{k}^{*}$ with subsequent extraction of the imaginary part allow to obtain the current conservation equation,

$$
\sum_{j=j(k)} I_{j k}=0 .
$$

The form of Eq. (13) coincides 2] with that of the Schrödinger equation for a single electron hopping between the sites of the lattice with the same geometry in the presence of external magnetic field. As a consequence, $T_{c}(f)$ can be related with $\epsilon_{0}(f)$, the largest eigenvalue of the Schrödinger equation in the same field. For a network whose nodes are all characterized by the same coordination number $z$ this relation can be written [5] as

$$
\frac{T_{c 0}-T_{c}(f)}{T_{c 0}}=\left[\frac{\bar{\xi}}{L} \arccos \frac{\epsilon_{0}(f)}{z}\right]^{2} .
$$

Starting from the work of Hofstadter 32] (who considered the case of a square lattice), the spectrum of the Schrödinger equation for a single electron hopping problem in the presence of external magnetic field has been extensively studied for various types of two-dimensional lattices including triangular [33], honeycomb 34], dice 22] and kagome 16, 35] lattices.

The structure of the superconducting state in the network just below $T_{c}(f)$ is determined by the structure of the eigenfunction corresponding to $\epsilon_{0}(f)[\underline{6}$. The conditions for the applicability of the mean field approach are discussed in Appendix A.

\section{A network with a dice lattice geometry}

Dice lattice [36, 37] is formed by the sites with the coordination numbers 3 and 6 in such a way that each bond connects two sites with different coordination numbers (see Fig. 1). Below, when discussing a dice lattice, we denote the three-fold coordinated sites $k$ and the sixfold coordinated sites $j$. Thus, the bond $(j k)$ of a dice lattice connects the six-fold coordinated site $j$ with the three-fold coordinated site $k$. 
When considering the problem on a dice lattice it is convenient to simplify the quadratic form (11) by minimizing it with respect to all variables $\Delta_{k}$ defined on the three-fold coordinated sites. Substitution [from Eq. [13)] of

$$
\Delta_{k}=\frac{1}{3 \cos \eta} \sum_{j=j(k)} \Delta_{j} e^{i A_{j k}}
$$

into Eqs. (91)-(11) then gives:

$$
\begin{aligned}
F_{\mathrm{nw}}^{(2)}= & \frac{F_{2}}{3 \cos \eta} \sum_{\left(j j^{\prime}\right)}\left[\left(3 \cos ^{2} \eta-1\right)\left(\left|\Delta_{j}\right|^{2}+\left|\Delta_{j^{\prime}}\right|^{2}\right)\right. \\
& \left.-2 \cos (\pi f)\left(e^{i A_{j j^{\prime}}} \Delta_{j} \Delta_{j^{\prime}}^{*}+\text { c.c. }\right)\right]
\end{aligned}
$$

where the summation is performed over the pairs $\left(j j^{\prime}\right)$ of nearest neighbors on the triangular lattice formed by the six-fold coordinated sites, whereas variables

$$
A_{j j^{\prime}}=\left[\left(A_{j k^{\prime}}+A_{k^{\prime} j^{\prime}}\right)+\left(A_{j k^{\prime \prime}}+A_{k^{\prime \prime} j^{\prime}}\right)\right] / 2
$$

(where $k^{\prime}$ and $k^{\prime \prime}$ are the two three-fold coordinated sites belonging to the same rhombus as $j$ and $j^{\prime}$ ) are the averages of $A_{j j^{\prime}}$ on the two shortest paths on a network connecting the nodes $j$ and $j^{\prime}$. It follows from Eq. (12) that the variables $A_{j j^{\prime}} \equiv-A_{j^{\prime} j}$ have to satisfy the constraint

$$
\sum_{\square} A_{j j^{\prime}}=3 \pi f
$$

on all plaquettes of the triangular lattice. The form of Eqs. (17) and (19) suggests that for $0 \leq f \leq 1 / 2$ the problem of finding $T_{c}(f)$ on a dice lattice is reduced to analogous problem on a triangular lattice with $f$ multiplied by $3 / 2$ and a different value of $\eta$. Accordingly, the relation between the critical temperatures (expressed in terms of $\eta$ ) in the two cases is given by

$$
3 \cos ^{2} \eta_{c}(f)-1=2 \cos (\pi f) \cos \eta_{c}^{\triangle}(3 f / 2) .
$$

Analogous relation between the single electron spectra on dice and triangular lattices has been derived in Ref. 22.

Quite remarkably, for $f=1 / 2$ the non-diagonal coupling in Eq. (17) completely disappears, which allows immediately to conclude that

$$
\eta_{c}(1 / 2)=\arccos (1 / \sqrt{3}) \approx 0.9553 .
$$

This absence of coupling between different variables $\Delta_{j}$ can be understood as a manifestation of the extremely localized nature of the highly degenerate eigenfunctions 22. corresponding to the largest eigenvalue of the single electron Hamiltonian.

As a consequence, for $f=1 / 2$ the value of $F_{\mathrm{nw}}^{(2)}$ turns out to be exactly the same for any set of the variables $\Delta_{j}$ satisfying the normalization condition

$$
\frac{1}{N} \sum_{j}\left|\Delta_{j}\right|^{2}=\Delta^{2}
$$

where $N$ is the number of the six-fold coordinated sites in the network with appropriately chosen periodic boundary conditions (the total number of sites being $3 N$ ). Accordingly, to find the structure of the superconducting state in a fully frustrated wire network with the dice lattice geometry (which is the main subject of this work) one has to minimize the fourth-order contribution to free energy,

$$
F_{\mathrm{nw}}^{(4)}=\sum_{(j k)} F_{\mathrm{wire}}^{(4)}\left(\Delta_{j}, \Delta_{k}, A_{j k}\right),
$$

[where $\Delta_{k}$ is given by Eq. [16]) with respect to the whole set of the variables $\Delta_{j}$ satisfying the constraint (22), which fixes also the value of $F_{\mathrm{nw}}^{(2)}$. For $0<\eta-\eta_{c} \ll 1$

$$
F_{\mathrm{nw}}^{(2)} \approx-12 N F_{2} \Delta^{2}\left(\sin \eta_{c}\right)\left(\eta-\eta_{c}\right),
$$

where we have kept only the lowest order term of the expansion with respect to $\eta-\eta_{c}$.

At the conceptual level, this task can be considered as analogous to finding the structure of the vortex lattice which minimizes the forth-order contribution to the free energy of a bulk superconductor just below $H_{c 2}$. In this problem (first analyzed by Abrikosov [29]), the harmonic contribution to free energy is degenerate with respect to a huge number of continuous variables, the positions of the order parameter singularities, whereas in the present problem a huge continuous degeneracy of the harmonic problem is related with variables $\Delta_{j}$.

\section{FOURTH-ORDER CONTRIBUTION TO FREE ENERGY}

\section{A. A single wire}

Substitution of Eq. (4) into Eq. (2) describing the fourth-order contribution to the free energy of a superconducting wire gives

$$
\begin{aligned}
F_{\text {wire }}^{(4)}\left(\Delta_{j}, \Delta_{k}, A_{j k}\right) & =F_{4}\left[I_{4} \cdot\left(\left|\Delta_{j}\right|^{4}+\left|\Delta_{k}\right|^{4}\right)\right. \\
& +2 I_{3} \cdot\left(\left|\Delta_{j}\right|^{2}+\left|\Delta_{k}\right|^{2}\right)\left\langle\Delta_{j} \mid \Delta_{k}\right\rangle \\
& \left.+I_{2} \cdot\left(2\left|\Delta_{j}\right|^{2}\left|\Delta_{k}\right|^{2}+\left\langle\Delta_{j} \mid \Delta_{k}\right\rangle^{2}\right)\right]
\end{aligned}
$$

where $F_{4}=\beta L / 4$ and the numerical constants $I_{n}$ (with $n=2,3,4)$ are given by the integrals

$$
I_{n}=\int_{0}^{1} d t \frac{\sin ^{n}(\eta t) \sin ^{4-n}[\eta(1-t)]}{\sin ^{4} \eta} .
$$

When we are interested in the structure of the superconducting phase just below $T_{c}(f)$, the comparison of the forth order terms in the free energy of different states should be made by calculating them at $T=T_{c}(f)$. Thus, in the following we will need the values of $I_{n}$ at $\eta=\eta_{c}(1 / 2)=\arccos (1 / \sqrt{3})$, which are

$$
\begin{aligned}
& I_{2}=\left(15-9 \sqrt{2} \eta_{c}{ }^{-1}\right) / 32 \approx 0.0524, \\
& I_{3}=\sqrt{3}\left(7 \sqrt{2} \eta_{c}{ }^{-1}-9\right) / 32 \approx 0.0737, \\
& I_{4}=\left(27-13 \sqrt{2} \eta_{c}{ }^{-1}\right) / 32 \approx 0.2424 .
\end{aligned}
$$




\section{B. A tripod of three wires}

For $f=1 / 2$ the contribution to $F_{\text {nw }}^{(4)}$ from a tripod formed by the three links $\left(j_{a} k\right)$ [where $j_{a} \equiv j_{a}(k)$ with $a=1,2,3$ are the three nearest neighbors of $k$ numbered in the positive direction] after the substitution of Eq. (16) can be rewritten as

$$
\begin{aligned}
& F_{\mathrm{nw}}^{(4)}(k)= \\
& F_{4}\left[\frac{3 \nu_{1}+\nu_{2}}{4} \sum_{a=1}^{3}\left|\Delta_{j_{a}}\right|^{4}+\frac{\nu_{1}+\nu_{2}}{4}\left(\sum_{a=1}^{3}\left|\Delta_{j_{a}}\right|^{2}\right)^{2}+\right. \\
& +\nu_{1}\left(\sum_{a=1}^{3}\left|\Delta_{j_{a}}\right|\left|\Delta_{j_{a+1}}\right| \sin \chi_{j_{a} j_{a+1}}\right)^{2}+ \\
& +\nu_{2} \sum_{a=1}^{3}\left(\left|\Delta_{j_{a}}\right|\left|\Delta_{j_{a+1}}\right| \sin \chi_{j_{a} j_{a+1}}\right)^{2}- \\
& -\nu_{1} \sum_{a=1}^{3}\left|\Delta_{j_{a}}\right|\left|\Delta_{j_{a+1}}\right|\left|\Delta_{j_{a+2}}\right|^{2} \sin \chi_{j_{a} j_{a+1}}- \\
& \left.-\left(\nu_{1}+\nu_{3}\right) \sum_{a=1}^{3}\left|\Delta_{j_{a}}\right|\left|\Delta_{j_{a+1}}\right|\left(\left|\Delta_{j_{a}}\right|^{2}+\left|\Delta_{j_{a+1}}\right|^{2}\right) \sin \chi_{j_{a} j_{a+1}}\right]
\end{aligned}
$$

where

$$
\begin{aligned}
& \nu_{1}=\frac{4}{3} I_{2}+\frac{16}{3 \sqrt{3}} I_{3}+\frac{4}{3} I_{4}, \\
& \nu_{2}=\frac{4}{3} I_{2}, \\
& \nu_{3}=\frac{8}{3} I_{2}+\frac{4}{\sqrt{3}} I_{3},
\end{aligned}
$$

whereas $\chi_{j j^{\prime}}$ are the gauge-invariant phase differences between the phases $\varphi_{j}$ of the order-parameter $\Delta_{j} \equiv\left|\Delta_{j}\right| \exp \left(i \varphi_{j}\right)$ at neighboring six-fold coordinated sites,

$$
\chi_{j j^{\prime}}=\varphi_{j^{\prime}}-\varphi_{j}-A_{j j^{\prime}} \equiv-\chi_{j^{\prime} j}
$$

It follows from Eq. (19) that for all tripods, or, in other words, for all plaquettes of the triangular lattice formed by the six-fold coordinated sites,

$$
\sum_{a=1}^{3} \chi_{j_{a} j_{a+1}}=-\sum_{a=1}^{3} A_{j_{a} j_{a+1}}=-3 \pi / 2
$$

Since each plaquette of this lattice has a particular threefold coordinated site in its center, the index $k$ numbering such sites can be also used for numbering triangular plaquettes.

In the cyclic sums in Eq. (28) and analogous sums below $j_{4} \equiv j_{1}$. The last term in Eq. (28) can be omitted, since during summation over the whole lattice the two tripods adjacent to any link $\left(j_{a} j_{b}\right)$ always yield opposite contributions.

\section{The equal amplitude hypothesis}

Let us now introduce the additional assumption (whose self-consistency is established in Appendix [B] that the absolute values of the variables $\Delta_{j}$ are the same for all six-fold coordinated sites $j$,

$$
\Delta_{j}=\Delta \exp \left(i \varphi_{j}\right)
$$

where $\Delta$ is real. In that case the contributions to

$$
F_{\mathrm{nw}}^{(4)}=\sum_{k} F_{\mathrm{nw}}^{(4)}(k)
$$

from the next but last term in Eq. (28) coming from the neighboring tripods also cancel each other, and the expression for $F_{\text {nw }}^{(4)}$ is reduced to

$$
F_{\mathrm{nw}}^{(4)}=F_{4} \Delta^{4} \sum_{k}\left[\nu_{0}+V\left(\left\{\chi_{j_{a} j_{a+1}}\right\}\right)\right]
$$

where $\nu_{0}=(9 / 2) \nu_{1}+3 \nu_{2}$,

$V(\{\chi\})=V\left(\chi_{1}, \chi_{2}, \chi_{3}\right) \equiv \nu_{1}\left(\sum_{a=1}^{3} \sin \chi_{a}\right)^{2}+\nu_{2} \sum_{a=1}^{3} \sin ^{2} \chi_{a}$

and

$$
\sum_{a=1}^{3} \chi_{j_{a} j_{a+1}}=\pi / 2(\bmod 2 \pi)
$$

Since $F_{\mathrm{nw}}^{(4)}$ is invariant with respect to the shift of any of the variables $\chi_{j j^{\prime}}$ by a multiple of $2 \pi$, here and below we for convenience assume that they all are reduced to the interval $[-\pi, \pi]$, in accordance with which the right hand side of Eq. (35) is written as $\pi / 2(\bmod 2 \pi)$ instead of $-3 \pi / 2$, as it would follow from Eq. (30).

At the temperature of the superconducting transition in a fully frustrated wire network [that is, at $\eta=\eta_{c}=$ $\left.\eta_{c}(1 / 2)\right]$ the values of the coefficients $\nu_{n}$ are given by

$$
\begin{aligned}
& \nu_{1}=\frac{1}{4}\left(1+\sqrt{2} \eta_{c}^{-1}\right) \approx 0.6201 \\
& \nu_{2}=\frac{1}{8}\left(5-3 \sqrt{2} \eta_{c}^{-1}\right) \approx 0.0699 \\
& \nu_{0}=3
\end{aligned}
$$

\section{MINIMIZATION OF THE FOURTH-ORDER CONTRIBUTION TO FREE ENERGY}

\section{A. A single triangle}

It is well known that the ground state [38, 39] of the antiferromagnetic $X Y$ model with triangular lattice can be found by minimizing the energy separately for each triangular plaquette and then matching these solutions with each other.

For $\nu_{1}, \nu_{2}>0$ the minimum of $V\left(\chi_{1}, \chi_{2}, \chi_{3}\right)$ on an isolated triangle [under the constraint of the form (35)] 
is achieved when two of the arguments of $V\left(\chi_{1}, \chi_{2}, \chi_{3}\right)$ are equal to each other, for example

$$
\chi_{1}=\chi_{2}=-\psi(t), \chi_{3}=\pi / 2+2 \psi(t),
$$

another solution with the same value of $V\left(\chi_{1}, \chi_{2}, \chi_{3}\right)$ being

$$
\chi_{1}=\chi_{2}=-[\pi-\psi(t)], \chi_{3}=\pi / 2-2 \psi(t),
$$

where $t=\nu_{2} / \nu_{1}$ and

$$
\psi(t)=\arcsin \frac{\sqrt{12+4 t+t^{2}}-2+t}{4(1+t)} .
$$

With increase of $t$ from zero to infinity $\psi(t)$ continuously increases from $\arcsin [(\sqrt{3}-1) / 2] \approx \pi / 8$ to $\pi / 6$.

In an infinite system each variable $\chi_{j j^{\prime}}$ belongs simultaneously to two triangles, but enters the function $V\left(\chi_{1}, \chi_{2}, \chi_{3}\right)$ on these two triangles with the opposite signs. Comparison of Eqs. (37) and (38) with each other allows to conclude that it is impossible to minimize $F_{\mathrm{nw}}^{(4)}$ by minimizing $V\left(\chi_{1}, \chi_{2}, \chi_{3}\right)$ separately for each triangle.

\section{B. A periodic solution}

When variables $\chi_{j j^{\prime}}$ are reduced to a finite interval, the average value of $\sum_{a=1}^{3} \chi_{j_{a} j_{a+1}}$ should be equal to zero. This can be achieved if on one quarter of triangles the right hand side of Eq. (35) is equal to $-3 \pi / 2$, whereas on all remaining triangles it is really equal to $\pi / 2$. Accordingly, the minimal supercell of a periodic set of variables $\chi_{j j^{\prime}}$ should consist of four triangles.

The four-triangle supercell with the most symmetric (triangular) shape, but with the most general structure allowing for construction of a periodic state by a periodic repetition of this supercell, is shown in Fig. 2a. It can be described by the six variables $\chi_{i}$ (with $\left.i=1, \ldots, 6\right)$ defined as shown in Fig. 2a and satisfying three independent constraints of the form (35), which can be chosen to be

$$
\begin{aligned}
\chi_{1}+\chi_{2}+\chi_{3} & =\pi / 2, \\
-\chi_{3}+\chi_{4}+\chi_{5} & =\pi / 2, \\
-\chi_{1}-\chi_{5}+\chi_{6} & =\pi / 2,
\end{aligned}
$$

the fourth constraint,

$$
-\chi_{2}-\chi_{4}-\chi_{6}=-3 \pi / 2
$$

following automatically from Eqs. (40)-(42).

The minimization of $F_{\mathrm{nw}}^{(4)}$ for this supercell with respect to the remaining three degrees of freedom shows that for $\chi_{i} \in[-\pi, \pi]$ the minimum is achieved when

$$
\begin{aligned}
& \chi_{1}=\chi_{4}=-\pi / 4, \quad \chi_{3}=0, \\
& \chi_{2}=\chi_{5}=3 \pi / 4, \quad \chi_{6}=\pi,
\end{aligned}
$$

(a)

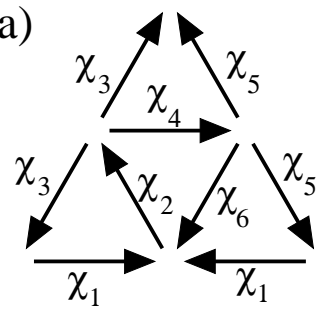

(b)
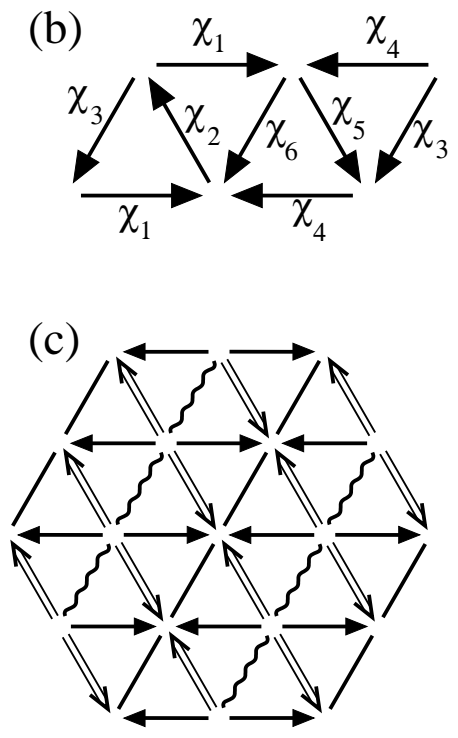

FIG. 2: Construction of periodic patterns minimizing the fourth-order contribution to free energy. (a) A possible choice for the most symmetric four triangle supercell. (b) An alternative four triangle supercell. (c) The structure of the periodic solution obtained from free energy minimization with the supercell shown in (a) or (b). Simple arrows correspond to phase differences $\chi_{j j^{\prime}}$ equal to $\pm \pi / 4$, double arrows to $\pm 3 \pi / 4$, simple lines to 0 and wiggly lines to $\pi$.

or in one of the five other states which can be constructed from this state by permutations of the variables $\chi_{i}$. In all these states on all triangles

$$
\sum_{a=1}^{3} \sin \chi_{j_{a} j_{a+1}}=0,
$$

which means that on each triangle the first term of $V(\{\chi\})$ reaches its absolute minimum (i.e., is equal to zero). Accordingly, the value of $F_{\mathrm{nw}}^{(4)}$ in these states does not depend on $\nu_{1}$,

$$
F_{\mathrm{nw}}^{(4)}=2\left(\nu_{0}+\nu_{2}\right) F_{4} \Delta^{4} N .
$$

Note that the supercell defined by Eqs. (44) consists of two pairs of equivalent (if one takes into account the equivalence of $\pi$ and $-\pi$ ) triangles. Thus the actual size of the supercell has turned out to be two times smaller than it has been initially conjectured. But there was no way to predict this without really performing the minimization for the four-triangle supercell. 
The same solution (whose structure is shown in Fig 2c) can be also found by starting from the assumption that a periodic state is formed with the help of the four-triangle supercell with the different shape shown in Fig. 2b. In that case the constraints (40) and (41) retain their form, whereas in Eqs. (42) and (43) one should interchange $\chi_{1}$ and $\chi_{4}$. For this supercell the minimum of $F_{\mathrm{nw}}^{(4)}$ (for not too large ratio $\left.\nu_{2} / \nu_{1}\right)$ is again achieved in the solution described by Eqs. (44) (or other equivalent solutions).

It follows from Eq. (16) that for $\left|\Delta_{j_{a}}\right|=\Delta$

$$
\left|\Delta_{k}\right|^{2}=\frac{\Delta^{2}}{3}\left(3-2 \sum_{a=1}^{3} \sin \chi_{j_{a} j_{a+1}}\right) .
$$

Substitution of Eq. (45) into Eq. (47) allows immediately to conclude that in the solution which we have found the absolute value of the order parameter on all three-fold coordinated sites has the same value as on the six-fold coordinated sites,

$$
\left|\Delta_{k}\right|=\left|\Delta_{j}\right|=\Delta
$$

In addition to gauge-invariant variables $\chi_{j j^{\prime}}$ defined on the bonds of triangular lattice, one can, naturally, also introduce the gauge-invariant phase differences defined on the bonds of the original dice lattice,

$$
\theta_{j k}=\varphi_{k}-\varphi_{j}-A_{j k}
$$

where $\varphi_{k} \equiv \arg \left(\Delta_{k}\right)$. As a consequence of Eq. (12), the variables $\theta_{j k}=-\theta_{k j}$, which we assume to be reduced to the interval $(-\pi, \pi)$, have to satisfy the constraint

$$
\sum_{\square} \theta_{j k}= \pm \pi
$$

When Eq. (48) is fulfilled, the expression (8) for the current in a link is reduced to

$$
I_{j k}=I_{0} \sin \theta_{j k}
$$

where

$$
I_{0}=\frac{2 e}{\hbar} \frac{\gamma \Delta^{2}}{\xi \sin \eta}
$$

Calculation of

$$
\Delta_{j} \Delta_{k}^{*} \exp \left(i A_{j k}\right)=\Delta^{2} \exp \left(-i \theta_{j k}\right)
$$

with the help of Eq. (16) demonstrates that in the considered state the variables $\theta_{j k}$ have the same three values (up to a permutation and a simultaneous change of sign),

$$
\theta_{j k}= \pm \theta_{1}, \pm \theta_{2}, \mp \theta_{3},
$$

on all tripods forming dice lattice. These values have to satisfy the constraints,

$$
\theta_{2}-\theta_{1}=\pi / 4, \quad \theta_{1}+\theta_{3}=\pi / 2, \quad \theta_{2}+\theta_{3}=3 \pi / 4,
$$

leading to the automatic fulfillment of Eqs. (50), as well as the current conservation equation,

$$
\sin \theta_{1}+\sin \theta_{2}=\sin \theta_{3}
$$

which follows from Eq. (51). As a consequence, they turn out to be exactly the same,

$$
\theta_{1,3}=\arccos (1 / \sqrt{3} \mp 1 / \sqrt{6}), \theta_{2}=\arccos (1 / \sqrt{3})
$$

as in the ground state of the fully frustrated $X Y$ model (FFXYM) with a dice lattice [26], for which the current conservation equation also has the form (56).

Thus, in terms of $\theta_{j k}$, the state which we have found (it is schematically shown in Fig. 3a) has exactly the same structure as one of the ground states of the FFXYM with a dice lattice. It has to be emphasized that the reasons for that are more subtle than a simple reduction of one model to the other. Firstly, in the case of a superconducting wire network the relation (48), the form of which seems to imply a possible reduction to $X Y$ model, is valid only in the minimum of free energy. Secondly, the substitution of Eq. (48) into Eq. (25) gives

$$
F_{\text {wire }}^{(4)}\left(\theta_{j k}\right)=F_{1}\left[2\left(I_{4}+I_{2}\right)+8 I_{3} \cos \theta_{j k}+4 I_{2} \cos ^{2} \theta_{j k}\right],
$$

whereas in the case of the FFXYM in the expression for the energy of a bond the term proportional to $\cos ^{2} \theta$ is simply absent, whereas the term proportional to $\cos \theta$ has a coefficient of the opposite (negative) sign. Thus the two models do not become equivalent even if Eq. (48) is artificially introduced as an additional assumption.

\section{Additional degeneracy}

The ground state of the FFXYM with a dice lattice is known to possess a well-developed accidental degeneracy, which can be described in terms of the formation of a network of zero-energy domain walls 26 ] on the background of a periodic state. This construction can start from any of the four periodic states shown in Fig. 3 and allows to obtain, in particular, the three other states shown in that figure by introduction of such domain walls.

For example, both state (c) and state (e) can be obtained from state (a) by introduction of a dense sequence of parallel zero-energy domain walls (of two different types). In that language state (g) can be described as the dense network of intersecting domain walls of two types. On the other hand, if one starts the construction from state (c), both state (a) and state (g) are formed by introduction of parallel domain walls, whereas the network of two types of walls corresponds to state (e).

The same set of states (described in a more detail in Ref. 26) minimizes $F_{\text {nw }}^{(4)}$ for given $\Delta$. In Appendix B we check that all these states are extremal not only when one assumes $\left|\Delta_{j}\right|=$ const, but also in the absence of this 

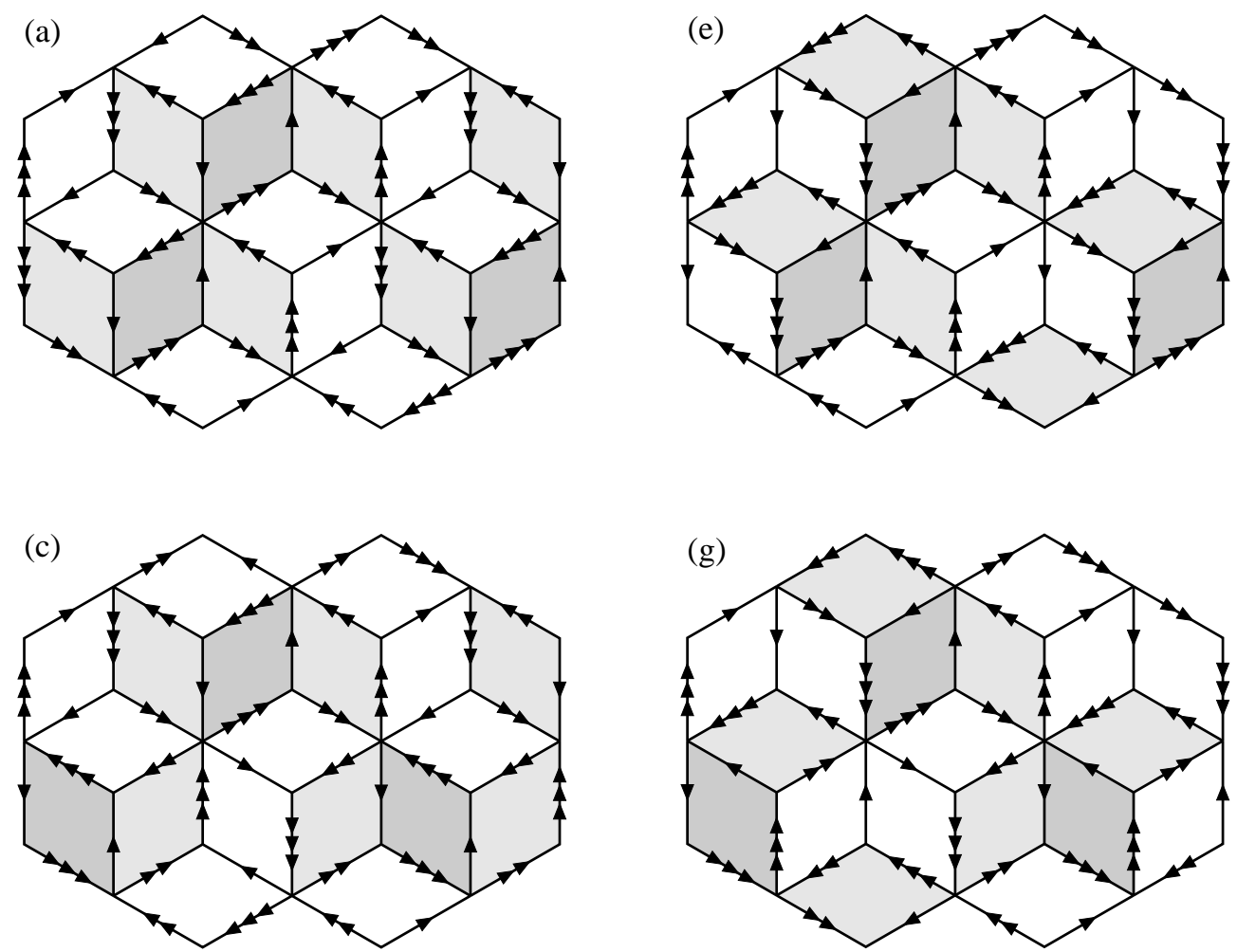

FIG. 3: The four periodic states generating the class of degenerate states discussed in the text by adjunction of domain walls. The plaquettes with positive vorticities are shaded, and the three types of arrows correspond to the three possible gauge-invariant phase differences $\theta_{1}, \theta_{2}, \theta_{3}$ (modulo $2 \pi$ ). Labels are chosen to match those introduced in Ref. 26 .

constraint. The accidental degeneracy related to formation of zero-energy domains walls gives rise to residual entropy proportional to the linear size of the system.

In the framework of the description of different states in a network in terms of the gauge-invariant phase differences $\theta_{j k} \in(-\pi, \pi)$, all rhombic plaquettes $\alpha$ of a dice lattice can be considered as occupied by positive and negative half-vortices, whose vorticities $m_{\alpha}= \pm 1 / 2$ are given by

$$
m_{\alpha}=\frac{1}{2 \pi} \sum_{\square} \theta_{j k}= \pm \frac{1}{2}
$$

In the family of states minimizing $F_{\mathrm{nw}}^{(4)}$ the half-vortices of the same sign always form triads with one "central" and two "edge" vortices [26]. The formation of domain walls which cost no free energy is related to the changes in the orientation and/or in the shape of these triads, but does not lead to formation of vortex clusters of any other size.

At low temperatures $\left(T \ll T_{c 0}\right)$ the free energy of a fully frustrated wire network with the dice lattice geometry (which then can be described in terms of the London approximation) is minimal for the same set of states, but with the slightly different values 26] of $\theta_{a}$

$$
\theta_{1}=\pi / 12, \quad \theta_{2}=\pi / 3, \quad \theta_{3}=5 \pi / 12,
$$

satisfying, nonetheless, the same constraints (55).

\section{Alternative solution}

The analysis of the supercell shown in Fig. $2 \mathrm{~b}$ allows also to find a state which minimizes $F_{\mathrm{nw}}^{(4)}$ for large $\nu_{2}$. In the notation of Fig. $2 \mathrm{~b}$ the structure of this state is given by

$$
\chi_{1}=\chi_{2}=\chi_{3}=-\chi_{4}=\pi / 6 ; \quad \chi_{5}=-\chi_{6}=5 \pi / 6 .
$$

It minimizes the value of the second term in Eq. (34) separately for each triangle, the value of $F_{\mathrm{nw}}^{(4)}$ being

$$
F_{\mathrm{nw}}^{(4)}=2\left[\nu_{0}+\frac{3}{4}\left(\nu_{1}+\nu_{2}\right)\right] F_{4} \Delta^{4} N .
$$

This alternative state is characterized by even more developed accidental degeneracy leading to extensive residual entropy. Namely, the value of $F_{\mathrm{nw}}^{(4)}$ does not change if at an arbitrary number of sites $j$ the variables $\varphi_{j}$ are shifted by $\pi$. Note that this property holds not only at $\nu_{1}=0$, when it trivially follows from $V(\{\chi\})$ being dependent only on $\cos 2 \chi_{a}$, but also at finite values of $\nu_{1}$.

Comparison of Eq. (62) with Eq. (46) shows that the values of $F_{\mathrm{nw}}^{(4)}$ in the two states become equal at $\nu_{2} / \nu_{1}=3$, whereas in our case, according to Eqs. (36), $\nu_{2} / \nu_{1} \approx 0.1127 \ll 3$. At so low values of the ratio $\nu_{2} / \nu_{1}$, the alternative solution discussed in this subsection is simply unstable. 


\section{MAGNETIC ENERGY}

\section{A. An arbitrary network}

When currents in a two-dimensional wire network satisfy the current conservation equations, the current in each link can be expressed as a difference of so called mesh currents [40], $I_{\alpha}^{m}$, associated with the plaquettes (meshes) of a network. Namely, the current in the link $(j k)$ can be written as the difference of the mesh currents associated with the two plaquettes $\left(\alpha\right.$ and $\left.\alpha^{\prime}\right)$ adjacent to this link,

$$
I_{j k}=I_{\alpha}^{m}-I_{\alpha^{\prime}}^{m} .
$$

The magnetic energy of the currents in the network, $E_{\text {magn }}$, can be then expressed in terms of $I_{\alpha}^{m}$,

$$
E_{\text {magn }}=\frac{1}{2 c^{2}} \sum_{\alpha, \beta} L_{\alpha \beta} I_{\alpha}^{m} I_{\beta}^{m},
$$

where a symmetric matrix $L_{\alpha \beta}$ is usually called the mutual inductance matrix [40.

The diagonal elements of this matrix describe the selfinductances of current loops which can be associated with different plaquettes of the network and, accordingly, have to be positive. On the other hand, its nondiagonal elements describe the mutual inductances of non-intersecting coplanar current loops. Magnetic fields of such loops substract from each other and, therefore, the non-diagonal elements of $L_{\alpha \beta}$ have to be negative. In an infinite network the constraint

$$
\sum_{\alpha} L_{\alpha \beta}=0
$$

has to be satisfied. This ensures the invariance of $E_{\text {magn }}$ with respect to a possible redefinition of mesh currents, $I_{\alpha}^{m} \rightarrow I_{\alpha}^{m}+\delta I^{m}$ that leaves the physical currents in the links, $I_{j k}$, intact. For practical purposes it is convenient to define $I_{\alpha}^{m}$ in such a way that

$$
\sum_{\alpha} I_{\alpha}^{m}=0 .
$$

The value of $L_{\alpha \beta}$ depends only on the relative disposition of the two plaquettes $\alpha$ and $\beta$, and in the limit of infinitely thin wires can be found by calculating the double integral over their perimeters $\Gamma_{\alpha}$ and $\Gamma_{\beta}$,

$$
L_{\alpha \beta}=\oint_{\Gamma_{\alpha}} d \mathbf{r}_{\alpha} \oint_{\Gamma_{\beta}} d \mathbf{r}_{\beta} \frac{1}{\left|\mathbf{r}_{\alpha}-\mathbf{r}_{\beta}\right|} .
$$

The expression for $L_{\alpha \beta}$ given by Eq. (67) in the case of $\alpha=\beta$ is logarithmically divergent, which means that $L_{\alpha \alpha}$ has always to be calculated more accurately, taking into account the finite width of the wires. The same is true for the value of $L_{\alpha \beta}$ for neighboring plaquettes (having a common link). In the case of more distant neighbors (having only a common node or simply not touching each other) one can use Eq. (67) based on the assumption of infinitely thin wires without encountering any divergences.

Eq. (67) can be also rewritten as the double integral over the areas of the plaquettes $\alpha$ and $\beta$. For $\alpha \neq \beta$

$$
L_{\alpha \beta}=-\int_{S_{\alpha}} d^{2} \mathbf{r}_{\alpha} \int_{S_{\beta}} d^{2} \mathbf{r}_{\beta} \frac{1}{\left|\mathbf{r}_{\alpha}-\mathbf{r}_{\beta}\right|^{3}},
$$

which shows that $\left|L_{\alpha \beta}\right|$ rapidly decays with the growth of $R_{\alpha \beta}$, the distance between the centers of the plaquettes $\alpha$ and $\beta$. For $R_{\alpha \beta} \gg L$

$$
L_{\alpha \beta} \approx-\frac{S_{\alpha} S_{\beta}}{R_{\alpha \beta}^{3}} .
$$

In any periodic state minimizing the free energy of a frustrated network, the plaquettes with negative and positive values of $I_{\alpha}^{m}$ regularly alternate with each other, so $E_{\text {magn }}$ (normalized, for example, per a single plaquette) is given by a rapidly decaying sign alternating lattice sum. It allows one to expect that the main contribution to this sum comes from its largest terms, corresponding to the self-inductances of the plaquettes and the mutual inductances of rather close neighbors. Analogously, when comparing the magnetic energies of different degenerate states minimizing the fourth order term in the free energy, the main contribution to the difference between them can be expected to come from the closest neighbors whose contributions do not cancel each other identically.

Besides the proper energy of the magnetic field induced by currents and given by Eq. (64), one also has to take into account the decrease of the superconducting free energy related to the vector potential of this field. In the weak screening regime the sum of these two contributions, $F_{\text {magn }}$, differs from $E_{\text {magn }}$ only by sign,

$$
F_{\text {magn }}=-E_{\text {magn }},
$$

and, therefore, one has to maximize $E_{\text {magn }}$.

\section{B. Mutual inductances of dice lattice plaquettes}

Fig. 4 introduces the classification of neighbors for rhombic plaquettes of a dice lattice, which can be used for the natural reordering of summation in Eq. (64). A chosen plaquette (which is shaded) has four nearest neighbors (denoted by 1 ), four next-to-nearest neighbors (denoted by 2), two third neighbors (denoted by 3 ), etc., as shown in Fig. 4 up to sixth neighbors. In the following we denote the self-inductance of a plaquette $L_{0}$ and the mutual inductance of a plaquette and its $n$-th neighbor (which is a negative quantity), $L_{n}=-\lambda_{n} L$.

For $n$ from 2 to 5 the calculation of $L_{n}$ with the help of Eq. (67) or Eq. (68) gives

$$
\begin{aligned}
\lambda_{2}= & 4 \sqrt{3}-2 \sqrt{7}-2-15 \ln 3-4 \ln (1+\sqrt{3}) \\
& +\ln \left[8(4+\sqrt{7})^{5}(-1+2 \sqrt{7})(1+25 \sqrt{7})^{2}\right],
\end{aligned}
$$




$$
\begin{aligned}
\lambda_{3}= & 8 \sqrt{7}-12 \sqrt{3} \\
& +\ln \left[(27 / 4)(1+\sqrt{3})^{12} /(5+2 \sqrt{7})^{6}\right], \\
\lambda_{4}= & 12-10 \sqrt{3}+2 \sqrt{7}-(33 / 2) \ln 2-(97 / 4) \ln 3 \quad \\
& +(1 / 2) \ln \left[(1+\sqrt{3})^{50}(4-\sqrt{7})^{31}(2+\sqrt{7})^{11}\right], \\
\lambda_{5}=3 & +5 \sqrt{3}-3 \sqrt{7}-\sqrt{13}-12 \ln 3 \\
& +\ln \left[(2+\sqrt{7})(1+2 \sqrt{7})(5+2 \sqrt{7})^{3} /(1+\sqrt{3})^{8}\right] \\
& +(1 / 2) \ln \left[(1+\sqrt{13})(-2+\sqrt{13})^{4}(7+\sqrt{13})^{8} / 2\right],
\end{aligned}
$$

whereas numerically

$$
\begin{aligned}
& \lambda_{2} \approx 0.5569, \\
& \lambda_{3} \approx 0.3637, \\
& \lambda_{4} \approx 0.1671 \\
& \lambda_{5} \approx 0.0723
\end{aligned}
$$

Thus the decrease of $\left|L_{n}\right|$ with $n$ is rather fast even for $n \sim 1$.

\section{The form and the magnitude of magnetic energy}

In a general situation the values of mesh currents $I_{\alpha}^{m}$ should be found from Eqs. (63) and, accordingly, are given by non-local linear combinations of link currents $I_{j k}$. Quite remarkably, substitution of Eq. (16) into Eq. (8) allows to find that the values of mesh currents in a fully frustrated superconducting wire network with dice lattice geometry in the vicinity of $T_{c}$ are given by the local expression,

$$
I_{j j^{\prime}}^{m}=-\frac{2 e}{\hbar} \frac{\gamma}{\xi \sin \eta}\left|\Delta_{j}\right|\left|\Delta_{j^{\prime}}\right| \frac{\cos \chi_{j j^{\prime}}}{\sqrt{3}},
$$

where $I_{j j^{\prime}}^{m}$ is the mesh current in the plaquette $\alpha_{j j^{\prime}}=\left(j k^{\prime} j^{\prime} k^{\prime \prime}\right)$.

In the family of states minimizing $F_{\mathrm{nw}}^{(4)}$ and described in Sec. IV the absolute value of $I_{j j^{\prime}}^{m}$ on all plaquettes

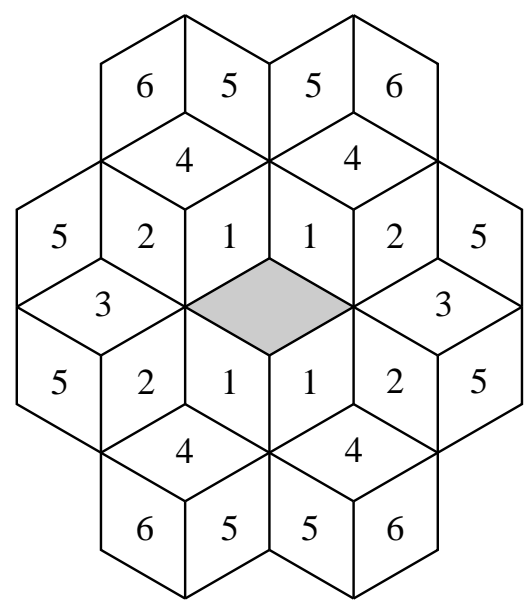

FIG. 4: Classification of plaquettes according to their distance from the shaded one. acquires only two values,

$$
I_{c}^{m}=I_{0} / \sqrt{3}, \quad I_{e}^{m}=I_{0} / \sqrt{6},
$$

where $I_{0}$ is given by Eq. (52). The choice between $I_{c}^{m}$ and $I_{e}^{m}$ is determined by whether the plaquette $\alpha_{j j^{\prime}}$ is occupied by the central or edge vortex of a triad to which it belongs, whereas the sign of $I_{j j^{\prime}}^{m}$ by the sign of this vortex. The ratio $g=I_{e}^{m} / I_{c}^{m}$ following from Eqs. (76) is equal to $g_{1}=1 / \sqrt{2}$.

Substitution of Eq. (75) into Eq. (64) shows that $E_{\text {magn }}$ is of the fourth order in $\Delta_{j}$, and thus should be added (with the negative sign) to the forth-order term, $F_{\mathrm{nw}}^{(4)}$, which has been minimized in Sec. IV. It follows from Eq. (75) that for $\left|\Delta_{j}\right|=\Delta$ the main contribution to $E_{\text {magn }}$ [which is related to self-inductances of lattice plaquettes, $L_{0} \sim 8 L \ln (L / d)$, where $d$ is the thickness of the wires], can be written as

$$
E_{\mathrm{magn}}^{(0)}=E_{\mathrm{m}} \sum_{\left(j j^{\prime}\right)}\left[1-\sin ^{2} \chi_{j j^{\prime}}\right]
$$

where $E_{\mathrm{m}}=L_{0} I_{0}^{2} / 6 c^{2}$.

The value of the coefficient $E_{\mathrm{m}}$, which at $T=T_{c}$ is given by

$$
E_{\mathrm{m}}=\frac{L_{0}}{4}\left(\frac{2 e}{\hbar c}\right)^{2}\left(\frac{\gamma}{\xi}\right)^{2} \Delta^{4}
$$

should be compared with $F_{4} \Delta^{4}=(L / 4) \beta \Delta^{4}$. With the help of Eq. A5 and Eq. A6 one obtains

$$
\frac{E_{\mathrm{m}}}{F_{4} \Delta^{4}} \sim \frac{\sigma}{\kappa^{2} \xi^{2}\left(T_{c}\right)},
$$

where $\kappa$ is the Ginzburg-Landau parameter of the material from which the wires are made, and $\sigma$ their crosssection area. Since the mean field phase transition in a fully frustrated network takes place when $\xi\left(T_{c}\right) \approx L$, Eq. (79) can be rewritten as

$$
\frac{E_{\mathrm{m}}}{F_{4} \Delta^{4}} \sim \frac{\sigma}{\kappa^{2} L^{2}} .
$$

Thus, in order to get $E_{\text {magn }} \ll F_{\text {nw }}^{(4)}$ (the weak screening regime) one should have

$$
\sigma \ll \kappa^{2} L^{2} .
$$

In other terms the same condition can be rewritten as

$$
\Lambda_{\mathrm{eff}}\left[\xi\left(T_{c}\right) \sim L\right] \gg L,
$$

where $\Lambda_{\text {eff }}(\xi) \sim(\kappa \xi)^{2} L / \sigma$ is the effective penetration depth for the magnetic field in a network. In such a case $E_{\text {magn }}$ can be treated as a small correction, which is relevant only for the removal of the accidental degeneracy between different states minimizing $F_{\mathrm{nw}}^{(4)}$. In the opposite limit finding the structure of the ordered state becomes an even more complicated problem because one has to 
minimize $F_{\mathrm{nw}}^{(2)}+F_{\mathrm{nw}}^{(4)}+E_{\text {magn }}$ taking into account the dependence of all three terms on the magnetic fields induced by the currents. Therefore, below we always assume $\sigma \ll \kappa^{2} L^{2}$, that is that the wires are thin enough.

Note, however, that the applicability of the mean field approach requires the wires to be not too thin, $\sigma \gg \kappa^{2} L^{3} / \Lambda_{\text {univ }}(T)$, see Appendix A. The two conditions on $\sigma$ are compatible provided $L \ll \Lambda_{\text {univ }}(T)$, which is readily satisfied in experiments, since for a temperature around one Kelvin $\Lambda_{\text {univ }}(T)$ is of the order of one centimeter, see Eq. A8.

It is clear from the form of Eq. (77) that the substraction of $E_{\text {magn }}^{(0)}$ from $F_{\text {nw }}^{(4)}$ does not change the functional form of the fourth order term (expressed in the terms of the variables $\chi_{j j^{\prime}}$ ), but leads only to a small increase of the coefficient $\nu_{2}$ in Eq. (34). The next contribution to $E_{\text {magn }}$, which is related to mutual inductances of neighboring plaquettes, has a more complicated structure,

$$
E_{\text {magn }}^{(1)}=-\frac{L_{1} I_{0}^{2}}{3} \sum_{k} \sum_{a=1}^{3} \cos \chi_{j_{a} j_{a+1}} \cos \chi_{j_{a+1} j_{a+2}} .
$$

However, in all the states minimizing $F_{\mathrm{nw}}^{(4)}$, the three variables $\chi_{j j^{\prime}}$ on each triangle are always given by

$$
\chi_{1,2,3}=\pi / 2 \mp \pi / 2, \mp \pi / 4, \pm 3 \pi / 4,
$$

as a consequence of which the sum over $a$ in Eq. (81) is equal to $-1 / 2$ for all $k$ and, therefore, the substraction of $E_{\text {magn }}^{(1)}$ from $F_{\mathrm{nw}}^{(4)}-E_{\text {magn }}^{(0)}$ also does not lift the degeneracy. To be sure of that we have checked also that all the states minimizing $F_{\mathrm{nw}}^{(4)}$ remain extremal with respect to variations of $\varphi_{j}$ even when one includes into analysis the extra terms obtained by the variation of $E_{\text {magn }}^{(1)}$.

\section{Selection of the state by magnetic energy}

In a more general situation, when the ratio $g=I_{e}^{m} / I_{c}^{m}$ is kept as a free parameter, the first two contributions to the magnetic energy can be written as

$$
\begin{aligned}
& E_{\text {magn }}^{(0)}=\frac{L_{0}}{2}\left(\frac{I_{c}^{m}}{c}\right)^{2}\left(1+2 g^{2}\right) N, \\
& E_{\text {magn }}^{(1)}=-L_{1}\left(\frac{I_{c}^{m}}{c}\right)^{2} 2 g^{2} N,
\end{aligned}
$$

and, naturally, are also the same for all the states minimizing $F_{\mathrm{nw}}^{(4)}$.

The difference appears when considering $E_{\text {magn }}^{(2)}$, the contribution to $E_{\text {magn }}$ coming from the mutual inductances of the plaquettes which are next-to-nearest neighbors of each other. Comparison of $E_{\text {magn }}^{(2)}$ for the four periodic states the structure of which is shown in Fig. 3 shows that for any $g$ with $|g|<1$ the minimum of $E_{\text {magn }}^{(2)}$ is achieved in the state (e) and the maximum in the state (c).
The non-equivalent contributions to magnetic energy of different periodic states can be characterized by the dimensionless parameter $\epsilon$ defined by the relation

$$
E_{\text {magn }}=E_{\text {magn }}^{(0)}+E_{\text {magn }}^{(1)}+\epsilon \frac{L\left(I_{c}^{m}\right)^{2}}{c^{2}} N .
$$

The values of $\epsilon$ for basic periodic states can be then expressed in terms of the coefficients $\lambda_{n}$ as

$$
\begin{aligned}
\epsilon_{a}= & 2 g^{2} \lambda_{2}-\left(1+2 g^{2}\right) \lambda_{3}+\left(2-4 g^{2}\right) \lambda_{4} \\
& +4 g^{2} \lambda_{5}+\ldots \\
\epsilon_{c}= & \left(1+g^{2}\right) \lambda_{2}-g^{2} \lambda_{3}+\left(1-3 g^{2}\right) \lambda_{4} \\
& -\left(2-2 g^{2}\right) \lambda_{5}+\ldots \\
\epsilon_{e}= & -2 g^{2} \lambda_{2}-\left(1-2 g^{2}\right) \lambda_{3}+2 \lambda_{4}-4 g^{2} \lambda_{5}+\ldots \\
\epsilon_{g}= & \left(1-3 g^{2}\right) \lambda_{2}+g^{2} \lambda_{3}+\left(1+g^{2}\right) \lambda_{4} \\
& -\left(2-2 g^{2}\right) \lambda_{5}+\ldots
\end{aligned}
$$

Substitution of the values of $\lambda_{n}$ given by Eqs. (74) into Eqs. (86)- (89) shows that for $|g|<1$ the state (c) maximizes the $n$-th approximation to $\epsilon$,

$$
\epsilon^{(n)}=\frac{c^{2}}{L\left(I_{c}^{m}\right)^{2} N} \sum_{m=2}^{n} E_{\text {magn }}^{(m)}
$$

not only for $n=2$ (as it follows from the previous paragraph), but also for all other values of $n$ it has been possible to check (up to $n=5$ ). Since the value of $\lambda_{n}$ rapidly decreases with the increase of $n$, the same conclusion can be expected to be valid in the limit of $n \rightarrow \infty$. Table 1 illustrates the dependence of $\epsilon^{(n)}$ on $n$ for four basic periodic states in the case of $g=1 / \sqrt{2}$.

In a Josephson junction array the value of the current in a junction is also given by Eq. (51), where now $I_{0}=(2 e / \hbar) J(J$ being the coupling constant of a single junction). As a consequence, the results of this section are applicable to a fully frustrated Josephson junction array as well. The value of the parameter $g$ in the array is also equal to $g_{1}$, because its ground state is characterized by exactly the same values of the variables $\theta_{a}$, Eq. (57), as in a fully frustrated wire network just below $T_{c}$.

On the other hand, for $T \ll T_{c 0}$, when $I_{j k} \propto \theta_{j k}$, the value of $g$ in a fully frustrated wire network is equal to $g_{0}=3 / 5$, as can be found from Eqs. (60). It can be expected that with the decrease in temperature the value of $g$ in a network continuously decreases from $g_{1}$ to $g_{0}$. However, the conclusion on the selection of the state (c) by the magnetic energy is valid not only for $g_{0} \leq g \leq g_{1}$, but in the whole interval $-1<g<1$.

\section{CONCLUSION}

The main result of this work is that the superconducting state in a fully frustrated wire network with the dice lattice geometry exhibits the same set of degenerate spacial patterns minimizing the free energy in the two limiting cases when the temperature is either low compared 
to the critical temperature $T_{c}$ (London limit) or close to $T_{c}$ (Ginzburg-Landau limit). This conclusion is quite interesting, given the fact that the system is described by two rather different models in these two limits. In the London limit, the amplitude fluctuations of the superconducting order parameter are negligible, and the corresponding fully frustrated $X Y$ model has been analyzed in [26]. In the vicinity of $T_{c}$, we used the variational approach pioneered by Abrikosov, where the spacial variations of the complex order parameter are constrained to live in the subspace of unstable modes for the corresponding linearized Ginzburg-Landau equations.

Our interest in this problem had been stimulated by the fact that for a fully frustrated network with the dice lattice geometry, this subspace has unusually high degeneracy (as a consequence of the localized nature of modes) and includes a finite fraction (one third) of the total number of modes 22, 30]. Remarkably, non linear effects select particular linear combinations of these spacially localized states which reproduce precisely the current patterns obtained for the pure $X Y$ limit [26].

In the second part of this article, we have compared magnetic energies of current patterns in different periodic states minimizing the Ginzburg-Landau functional. The dominant contribution to this degeneracy lifting mechanism is due to interactions of current loops which can be associated with second neighbor plaquettes. It favors the periodic pattern in which the triads of positive and negative vortices have three different orientations [state (c) in Fig. 3. The same conclusion is valid also in London limit and in the case of a Josephson junction array with the same geometry. It can be hoped that decoration experiments performed in more equilibrium conditions than those of Refs. 24, 25 may reveal such an ordering.

This work leaves several open questions. First, it is important to know if all the states constructed here are stable with respect to local fluctuations in both order parameter amplitudes and phases. We have checked numerically that this is indeed the case for the periodic states shown on Fig. 3 as well as for configurations with a single domain wall between two such ordered states. Unfortunately, a simple (analytical) stability proof holding for the complete class of degenerate states is not available now.

Second, alternative degeneracy lifting mechanisms should be analyzed, as has been done for a wire network

\begin{tabular}{c||c|c|c|c|}
$\mathrm{n}$ & $\mathrm{a}$ & $\mathrm{c}$ & $\mathrm{e}$ & $\mathrm{g}$ \\
\hline \hline 2 & 0.5569 & 0.8354 & -0.5569 & -0.2785 \\
\hline 3 & -0.1704 & 0.6536 & -0.5569 & -0.0966 \\
\hline 4 & -0.1704 & 0.5700 & -0.2227 & 0.1540 \\
\hline 5 & -0.0258 & 0.4977 & -0.3673 & 0.0817
\end{tabular}

TABLE I: Comparison of dimensionless parameter $\epsilon$ characterizing the magnetic energy of four basic periodic states in different orders of approximation. with kagome geometry by Park and Huse [20]. These authors have found that the dominant perturbation to the idealized Ginzburg-Landau description of a wire network arises from the finite width of wires, which removes the degeneracy between the two opposite orientations of the supercurrents in a given loop. This mechanism can be interpreted in the terms of magnetic field redistribution between the network plaquettes (see discussion in Ref. 41, where it has been named "hidden incommensurability"), and, accordingly, is effective only when a network contains non-equivalent plaquettes. Therefore, in the case of a dice lattice (formed by identical rhombic plaquettes) it cannot play a prominent role.

Another degeneracy lifting mechanism is related with the free energy of fluctuations around various free-energy minima and will be the subject of a separate report. It is likely to be the dominant one in the vicinity of $T_{c}$, but with decrease in temperature becomes less and less important in comparison with magnetic interactions of currents analyzed in this work.

\section{Acknowledgments}

The work of S.E.K. has been additionally supported by the Program "Quantum Macrophysics" of the Russian Academy of Sciences and by the Program "Scientific Schools of the Russian Federation" (grant No. 1715.2003.2).

\section{APPENDIX A: CONDITION FOR THE APPLICABILITY OF THE MEAN FIELD APPROACH}

In two-dimensional superconductors the most important fluctuations are the fluctuations of the order parameter phase $\varphi=\arg (\Delta)$. When $T_{c}$ is approached from below, the free energy of the phase fluctuations in a single wire,

$$
F_{\text {wire }} \approx \frac{J}{2}\left(\delta \varphi_{j}-\delta \varphi_{k}\right)^{2}
$$

can be characterized by the effective coupling constant $J$, which in the absence of external magnetic field is given by

$$
J(T)=\frac{\alpha(T) \gamma}{L \beta}=\frac{\gamma^{2}}{L \beta \xi^{2}(T)} .
$$

The fluctuations are of no importance when $J(T) \gg k_{B} T$, which means that the critical region corresponds to

$$
\xi^{2}(T) \gtrsim \frac{\gamma^{2}}{L \beta k_{B} T_{c 0}} .
$$


Since the maximal deviation of $T_{c}(f)$ from $T_{c 0}$ is achieved when $\xi\left[T_{c}(f)\right] \sim L[2,5]$, the critical region can be considered as sufficiently narrow if

$$
L^{3} \ll \frac{\gamma^{2}}{\beta k_{B} T_{c 0}} .
$$

When the mean free path of electron is much smaller than the thickness of a wire, the values of the coefficients entering the Ginzburg-Landau functional for the wire are determined simply by the values of the analogous coefficients for the material from which the wire is fabricated. Substitution of

$$
\beta \approx \sigma \beta_{\text {bulk }}, \quad \gamma \approx \sigma \gamma_{\text {bulk }},
$$

where $\sigma$ is the cross section of a wire, and

$$
\beta_{\mathrm{bulk}}=\frac{16 \pi^{3}}{\Phi_{0}^{2}} \gamma_{\mathrm{bulk}}^{2} \kappa^{2}
$$

where $\kappa$ is the ratio of the penetration depth and the coherence length, into Eq. (A4) allows one to rewrite this condition as

$$
L^{3} \ll \frac{\Lambda_{\text {univ }}\left(T_{c 0}\right) \sigma}{\pi \kappa^{2}},
$$

where

$$
\Lambda_{\text {univ }}(T)=\frac{\Phi_{0}^{2}}{16 \pi^{2} k_{B} T} \approx \frac{2 \mathrm{~cm} \cdot \mathrm{K}}{T}
$$

is the expression for the universal value of the twodimensional penetration length at the temperature of the Berezinskii-Kosterlitz-Thouless phase transition in a two-dimensional superconductor 42. In the right-hand side of Eq. (A8) the temperature should be expressed in Kelvin.

In aluminum wire networks fabricated with electron beam lithography $[4,5,8,14,23,24] T_{c 0} \approx 1.2 \mathrm{~K}$, and therefore $\Lambda_{\text {univ }}\left(T_{c 0}\right) \approx 1.7 \mathrm{~cm}$, whereas (according to the estimates of Park and Huse [20]) $\kappa \sim 1$.

\section{APPENDIX B: CONSISTENCY OF THE EQUAL AMPLITUDE HYPOTHESIS}

We shall now check that for the class of states described in section IV] in which the amplitudes $\left|\Delta_{j}\right|$ are the same for all six-fold coordinated sites $j$, the derivatives $\partial F_{\mathrm{nw}}^{(4)} / \partial\left|\Delta_{j}\right|$ are independent of the sixfold coordinated site $j$. If this property is satisfied, it is then possible to enforce the equilibrium condition $\partial\left[F_{\mathrm{nw}}^{(2)}+F_{\mathrm{nw}}^{(4)}\right] / \partial\left|\Delta_{j}\right|=0$ with equal amplitudes $\left|\Delta_{j}\right|$ since $F_{\mathrm{nw}}^{(2)}$ is proportional to $\sum_{j}\left|\Delta_{j}\right|^{2}$ (with a negative coefficient below the critical temperature of the network).

Derivation of $F_{\mathrm{nw}}^{(4)}=\sum_{k} F_{\mathrm{nw}}^{(4)}(k)$ with respect to $\left|\Delta_{j}\right|$ gives

$\frac{\partial F_{\mathrm{nw}}^{(4)}}{\partial\left|\Delta_{j}\right|}=F_{4} \Delta^{3}\left[8 \nu_{0}+4 \nu_{2} \sum_{b=1}^{6} \sin ^{2} \chi_{j j_{b}}-2 \nu_{1} \sum_{b=1}^{6} \sin \chi_{j_{b} j_{b+1}}\right]$,

where $j_{b}$ (with $b=1, \ldots, 6$ ) are the six neighbors of $j$ numbered in positive direction (see Fig. (5), and it is assumed that $j_{7} \equiv j_{1}$. The contribution from the third term in Eq. (28) vanishes from Eq. (B1) as a consequence of Eq. (45), which is valid for any tripod in any of the states discussed in section IV]

It turns out that the first sum in Eq. (B1) is equal to 2 for any $j$ in any of the considered degenerate states. On the other hand, summation of Eq. (45) over the six tripods containing the given site $j$ allows one to conclude that the second sum in Eq. (B1) is always equal to zero. Thus, the expression in the right hand side of Eq. (B1) does not depend on $j$ for any configuration with $\left|\Delta_{j}\right|=$ const described in section IV

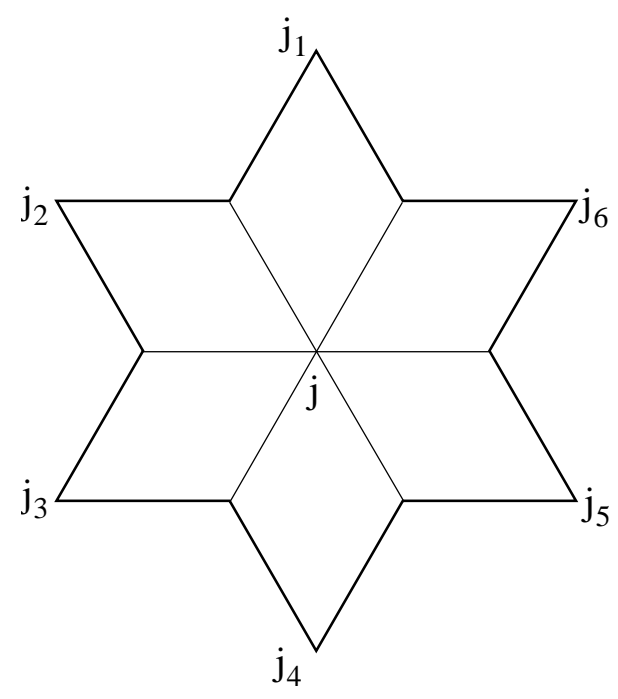

FIG. 5: The six neighbors of the site $j$ contributing to the sums in Eq. B1. 
[1] P.G. de Gennes, C. R. Sceances Acad. Sci., Ser. 2, 292, 9 and 291 (1981).

[2] S. Alexander, Phys. Rev. B 27, 1541 (1983).

[3] S. Alexander and E. Halevi, J. Phys. (Paris) 44, 805 (1983).

[4] B. Pannetier, J. Chaussy and R. Rammal, J. Phys. (Paris) 44, L853 (1983).

[5] B. Pannetier, J. Chaussy, R. Rammal and J.C. Villegier, Phys. Rev. Lett. 53, 1845 (1984).

[6] Y.Y.Wang, R. Rammal and B. Pannetier, J. Low Temp. Phys. 68, 301 (1987).

[7] J. Simonin and A. López, Phys. Rev. Lett. 56, 2649 (1986).

[8] J.M. Gordon, A.M. Goldman, J. Maps, D. Costello, R. Tiberio and B. Whitehead, Phys. Rev. Lett. 56, 2280 (1986); J.M. Gordon, A.M. Goldman and B. Whitehead, Phys. Rev. Lett. 59, 2311 (1987); J.M. Gordon and A.M. Goldman, Phys. Rev. B 35, 4909 (1987).

[9] F. Nori and Q. Niu, Phys. Rev. B 37, 2364 (1988); Q. Niu and F. Nori, Phys. Rev. B 39, 2134 (1989).

[10] S. Teitel and C. Jayaprakash, Phys. Rev. B 27, 598 (1983).

[11] S. Teitel and C. Jayaprakash, J. Phys. Lett. (Paris), 46, L33 (1985).

[12] W.Y. Shih and D. Stroud, Phys. Rev. B 32, 158 (1985).

[13] S.E. Korshunov, J. Stat. Phys. 43, 17 (1986).

[14] Yi Xiao, D.A. Huse, P.M. Chaikin, M.J. Higgins, S. Bhattacharya and D. Spencer, Phys. Rev. B 65, 214503 (2002).

[15] Y.-L. Lin, and F. Nori, Phys. Rev. B 65, 214504 (2002).

[16] Y.-L. Lin and F. Nori, Phys. Rev. B 50, 15953 (1994).

[17] D.A. Huse and A.D. Rutenberg, Phys. Rev. B 45, 7536 (1992).

[18] M.S. Rzchowski, Phys. Rev. B 55, 11745 (1997).

[19] M.J. Higgins, Yi Xiao, S. Bhattacharya, P.M. Chaikin, S. Sethuraman, R. Bojko and D. Spencer, Phys. Rev. B 61, R894 (2000)

[20] K. Park and D.A. Huse, Phys. Rev. B 64, 134522 (2001).

[21] S.E. Korshunov, Phys. Rev. B 65, 054416 (2002)
[22] J. Vidal, R. Mosseri and B. Douçot, Phys. Rev. Lett. 81, 5888 (1998).

[23] C.C. Abilio, P. Butaud, Th. Fournier, B. Pannetier, J. Vidal, S. Tedesco and B. Dalzotto, Phys. Rev. Lett. 83, 5102 (1999).

[24] B. Pannetier, C. C. Abilio, E. Serret, T. Fournier, P. Butaud, and J. Vidal, Physica C 352, 41 (2001).

[25] E. Serret, P. Butaud, and B. Pannetier, Europhys. Lett. 59, 225 (2002).

[26] S.E. Korshunov, Phys. Rev. B 63, 134503 (2001).

[27] V. Cataudella and R. Fazio, Europhys. Lett. 61, 341 (2003).

[28] V. Elser, Phys. Rev. Lett. 62, 2405 (1989).

[29] A.A. Abrikosov, Zh. Eksp. Teor. Fiz. 32, 1442 (1957) [Sov. Phys. - JETP 5, 1174 (1957)].

[30] J. Vidal, P. Butaud, B. Douçot, and R. Mosseri, Phys. Rev. B 64, 155306, (2001).

[31] C. Naud, G. Faini, and D. Mailly, Phys. Rev. Lett. 86, 5104, (2001).

[32] D.R. Hofstadter, Phys. Rev. B 14, 2239 (1976).

[33] F.H. Claro and G.H. Wannier, Phys. Rev. B 19, 6068 (1979).

[34] R. Rammal, J. Phys. (Paris) 46, 1345 (1985).

[35] Yi Xiao, V. Pelletier, P.M. Chaikin and D.A. Huse, Phys. Rev. 67, 104505 (2003).

[36] T. Horiguchi and C.C. Chen, J. Math. Phys. 15, 659 (1974).

[37] B. Sutherland, Phys. Rev. B 34, 5208 (1986).

[38] D.H. Lee, R.G. Caflisch, J.D. Joannopoulos and F.Y. Wu, Phys. Rev. B 29, 2680 (1984);

[39] S. Miyashita and J. Shiba, J. Phys. Soc. Jpn. 53, 1145 (1984).

[40] C.A. Desoer and E.S. Kuh, Basic Circuit Theory (McGraw-Hill, New York, 1969).

[41] R. Meyer, S.E. Korshunov, Ch. Leemann and P. Martinoli, Phys. Rev. B 66, 104503 (2002).

[42] M.R. Beasley, J.E. Mooij and T.P. Orlando, Phys. Rev. Lett. 42, 1165 (1979). 